Article

\title{
Preparation of Temozolomide-Loaded Nanoparticles for Glioblastoma Multiforme Targeting-Ideal Versus Reality
}

\author{
Chooi Yeng Lee ${ }^{1, *}$ and Ing Hong Ooi ${ }^{2}$ \\ 1 School of Pharmacy, Monash University Malaysia, Bandar Sunway, Selangor 47500, Malaysia \\ 2 Department of Pharmaceutical Chemistry, School of Pharmacy, International Medical University, \\ Kuala Lumpur 57000, Malaysia; inghong_ooi@imu.edu.my \\ * Correspondence: chooi.yeng.lee@monash.edu; Fax: +603-5514-6364 \\ Academic Editors: Alexandru Mihai Grumezescu and Vladimir P. Torchilin \\ Received: 13 July 2016; Accepted: 24 August 2016; Published: 8 September 2016
}

\begin{abstract}
Temozolomide (TMZ) is one of the most effective chemotherapeutic agents for glioblastoma multiforme, but the required high administration dose is accompanied by side effects. To overcome this problem and to further improve TMZ's efficacy, targeted delivery of TMZ by using polymeric nanoparticles has been explored. We synthesised the PLGA-PEG-FOL copolymer and attempted encapsulation of TMZ into PLGA-PEG-FOL nanoparticles using the emulsion solvent evaporation method and the nanoprecipitation method. Conjugation of PEG and FOL to PLGA has been reported to be able to increase the delivery of TMZ to the brain as well as targeting the glioma cells. However, despite making numerous modifications to these methods, the loading of TMZ in the nanoparticles only ranged between $0.2 \%$ and $2 \%$, and the nanoparticles were between $400 \mathrm{~nm}$ and $600 \mathrm{~nm}$ in size after freeze-drying. We proceed with determining the release profile of TMZ in phosphate buffered saline (PBS). Our initial data indicated that TMZ was slowly released from the nanoparticles. The metabolite of TMZ rather than the parent compound was detected in PBS. Our study suggests that while PLGA-PEG-FOL can be used as a polymeric or encapsulation material for central delivery of TMZ, a practical and cost effective formulation method is still far from reach.
\end{abstract}

Keywords: glioblastoma; polymeric nanoparticles; sustained release; targeted delivery; temozolomide

\section{Introduction}

Glioblastoma multiforme (GBM) is the most common primary brain tumour. It is classified by the World Health Organisation as a grade IV tumour. The current standard treatment for GBM begins with a maximal safe surgical resection, followed by a combined radiotherapy and temozolomide (Temodal ${ }^{\circledR}$, TMZ), and adjuvant TMZ therapy. The treatment regimen is by far the most effective, as it increases the median overall survival to 14.6 months. The percentage of patients alive at two years has also increased from $10.4 \%$ to $26.5 \%$ [1].

Temozolomide is a second-generation imidazotetrazine pro-drug, which has been shown in various studies to be an effective alkylating agent $[2,3]$. At physiological $\mathrm{pH}, \mathrm{TMZ}$ is metabolised to its active metabolite 5-(3-methyl-1-triazenyl)-imidazole-4-carboxamide (MTIC) [4]. Although TMZ has almost $100 \%$ bioavailability, due to its short half-life, high dose administration of TMZ is required and this leads to several dose-limiting side effects $[3,5,6]$. As a means to improve the efficacy of TMZ and lower the risk of side effects, targeted delivery such as the use of nanoparticles has been recommended $[5,7]$.

We used poly(lactic-co-glycolic acid) (PLGA) as our core material in nanoparticle formulation. PLGA is biodegradable and its hydrolytic products, lactic acid and glycolic acid, are effectively 
eliminated from the body as carbon dioxide and water [8,9]. We attempted to further increase the specificity of the nanoparticles by functionalising the nanoparticles with poly(ethylene) glycol (PEG) and folic acid (FOL). PEG increases the blood residence time of nanoparticles [10], while FOL has high affinity and specificity towards folate receptors [11]. Over-expression of folate receptors is often found on tumour cells, including glioma [12]. Cellular uptake of polymeric nanoparticles conjugated with FOL is 6.7 to 20 times higher than that of non-conjugated nanoparticles $[13,14]$. The fabrication and the use of PLGA-PEG-FOL as a carrier for anti-cancer drug is not new [15]. We successfully synthesised the PLGA-PEG-FOL copolymer by following the reported procedures with slight modification, and used the copolymer to encapsulate TMZ forming TMZ-loaded nanoparticles. The study investigated various conditions of two commonly used methods in the preparation of TMZ-loaded FOL-linked, PEGylated-PLGA nanoparticles. We then determined the percentage of TMZ loading in the nanoparticles and the size of the nanoparticles.

\section{Results}

The formulation and characteristics of TMZ-loaded PLGA-PEG-FOL nanoparticles prepared using the emulsion solvent evaporation method (Method 1) are shown in Table 1. In this method, the use of DCM as the organic phase yielded TMZ-loaded PLGA-PEG-FOL nanoparticles with a DL of $1 \%$, and the particle size was approximately $420 \mathrm{~nm}$. Increasing the amount of TMZ to $2.5 \mathrm{mg}$ from $2 \mathrm{mg}$ reduced the DL tremendously by 10 -fold. Suspending $5 \mathrm{mg}$ of TMZ in $2 \mathrm{~mL}$ of DCM increased the DL to $2 \%$, but the yield of the formulation was only $40 \%$ (Table 1 ). From the second solvent evaporation method, the DL was only $0.2 \%$. However, this method increased the yield to $68 \%$, and the particle size was reduced to less than $400 \mathrm{~nm}$ (Table 2). It should be noted that the sizes of the nanoparticles presented in Tables 1 and 2 were measured after freeze-drying.

Table 1. The preparation and the properties of TMZ-loaded nanoparticles developed using the solvent evaporation method.

\begin{tabular}{|c|c|c|c|c|c|c|c|}
\hline Preparation * & $\begin{array}{c}\text { TMZ } \\
\text { Amount } \\
(\mathrm{mg})\end{array}$ & $\begin{array}{l}\text { Solvent and } \\
\text { Volume }\end{array}$ & Yield (\%) & EE (\%) & DL (\%) & $\begin{array}{c}\text { Particle } \\
\text { Size } \pm \text { SD (nm) }\end{array}$ & $\begin{array}{c}\text { Zeta } \\
\underset{(\mathrm{mV})}{\text { Potential }} \pm \mathrm{SD}\end{array}$ \\
\hline $1^{\wedge}$ & 2 & $4 \mathrm{~mL} \mathrm{DCM}$ & $54.2 \pm 4.9$ & $11.90 \pm 4.4$ & $1.05 \pm 0.5$ & $424 \pm 94$ & $-20.8 \pm 13.6$ \\
\hline 3 & 5 & $2 \mathrm{~mL}$ DCM & 39.9 & 12.60 & 2.00 & $416 \pm 21$ & $-15.5 \pm 5.3$ \\
\hline
\end{tabular}

Table 2. The preparation and the property of TMZ-loaded nanoparticles developed using the solvent evaporation method, and with DMF used as the organic phase.

\begin{tabular}{|c|c|c|c|c|c|c|c|}
\hline Preparation * & $\begin{array}{c}\text { TMZ } \\
\text { Amount } \\
(\mathrm{mg})\end{array}$ & $\begin{array}{l}\text { Solvent and } \\
\text { Volume }\end{array}$ & Yield (\%) & EE (\%) & DL (\%) & $\begin{array}{c}\text { Particle } \\
\text { Size } \pm \text { SD }(\mathrm{nm})\end{array}$ & $\begin{array}{c}\text { Zeta } \\
\text { Potential } \pm S D \\
(\mathrm{mV})\end{array}$ \\
\hline $1^{\wedge}$ & 20 & $1 \mathrm{~mL}$ DMF & $67.90 \pm 17.40$ & $0.29 \pm 0.06$ & $0.19 \pm 0.18$ & $394 \pm 26$ & $-37.3 \pm 1.98$ \\
\hline
\end{tabular}

Table 3 is the summary of the formulation and characteristics of TMZ-loaded PLGA-PEG-FOL nanoparticles prepared using emulsion solvent evaporation involving dual solvent and two-phase TPGS solution (Method 3). 
Table 3. The preparation and the properties of TMZ-loaded nanoparticles developed using dual solvent and two-phase TPGS solution.

\begin{tabular}{|c|c|c|c|c|c|c|c|c|c|}
\hline Preparation * & Solvent $(2 \mathrm{~mL})$ & TPGS $1(2 \mathrm{~mL})$ & TPGS $2(15 \mathrm{~mL})$ & Yield (\%) & EE (\%) & DL (\%) & $\begin{array}{c}\text { Particle Size } \pm \text { SD (nm) } \\
\text { before Freeze-Drying }\end{array}$ & $\begin{array}{l}\text { Particle Size } \pm \text { SD (nm) } \\
\text { after Freeze-Drying }\end{array}$ & $\begin{array}{c}\text { Zeta Potential } \pm \text { SD } \\
(\mathrm{mV})\end{array}$ \\
\hline 1 & $\begin{array}{l}\text { DMSO:DCM } \\
0.5: 1.5\end{array}$ & $0.2 \%$ in water & $0.025 \%$ in water & 70.2 & 0.27 & 0.19 & $310.90 \pm 9.76$ & $473.10 \pm 48.5$ & $-56.76 \pm 1.06$ \\
\hline 2 & $\begin{array}{l}\text { DMF:DCM } \\
0.6: 1.4\end{array}$ & $0.2 \%$ in water & $0.025 \%$ in water & 83 & 0.26 & 0.13 & $146.60 \pm 0.14$ & $374.30 \pm 14.71$ & $-35.15 \pm 0.071$ \\
\hline 3 & $\begin{array}{l}\text { DMF:DCM } \\
\text { 0.6:1.4 }\end{array}$ & $\begin{array}{l}0.2 \% \text { in ethanol:water } \\
\text { (85:15) sat. with NaCI }\end{array}$ & $\begin{array}{c}0.025 \% \text { in } \\
\text { ethanol:water (85:15) } \\
\text { sat. with NaCI }\end{array}$ & 88.5 & 0.32 & 0.14 & $157.85 \pm 4.17$ & $520.50 \pm 21.28$ & $-56.65 \pm 0.212$ \\
\hline
\end{tabular}

${ }^{*}$ All preparations used $20 \mathrm{mg}$ TMZ, and $50 \mathrm{mg}$ PLGA-PEG-FOL copolymer. NaCI: sodium chloride.

Table 4. The preparation and the properties of TMZ-loaded nanoparticles developed using the nanoprecipitation method.

\begin{tabular}{|c|c|c|c|c|c|c|c|c|}
\hline Preparation *, & External Phase (15 mL) & $\begin{array}{c}\text { Saturation with } \\
\text { NaCI }\end{array}$ & Yield (\%) & EE (\%) & DL (\%) & $\begin{array}{c}\text { Particle Size } \pm \text { SD }(\mathrm{nm}) \\
\text { before Freeze-Drying }\end{array}$ & $\begin{array}{c}\text { Particle Size } \pm \text { SD (nm) } \\
\text { after Freeze-Drying }\end{array}$ & $\begin{array}{c}\text { Zeta Potential } \pm \text { SD } \\
(\mathrm{mV})\end{array}$ \\
\hline 1 & Water & No & $69.85 \pm 1.62$ & $0.32 \pm 0.03$ & $0.045 \pm 0.01$ & $187.55 \pm 0.21$ & $454.75 \pm 5.20$ & $-39.6 \pm 0.42$ \\
\hline 2 & Ethanol & No & $50.70 \pm 9.90$ & $0.31 \pm 0.12$ & $0.065 \pm 0.01$ & Not measured & $501.70 \pm 55.58$ & $-35.9 \pm 1.84$ \\
\hline 3 & Water & Yes & $87.85 \pm 3.32$ & $0.35 \pm 0.10$ & $0.035 \pm 0.01$ & $199.45 \pm 0.35$ & $511.50 \pm 37.05$ & $-40.0 \pm 0.21$ \\
\hline 4 & Ethanol:Water (50:50) & Yes & $88.20 \pm 2.12$ & $0.19 \pm 0.02$ & $0.021 \pm 0.001$ & $180.60 \pm 12.30$ & $501.20 \pm 9.48$ & $-40.2 \pm 11.3$ \\
\hline 5 & Ethanol:Water (85:15) & Yes & $92.30 \pm 0.42$ & $1.15 \pm 0.07$ & $0.170 \pm 0.01$ & $164.30 \pm 3.82$ & $436.50 \pm 37.50$ & $-47.0 \pm 2.69$ \\
\hline 6 & Ethanol:Water (95:5) & Yes & $58.35 \pm 7.14$ & $0.39 \pm 0.06$ & $0.075 \pm 0.01$ & $150.45 \pm 2.62$ & $632.5 \pm 58.69$ & $-47.3 \pm 6.29$ \\
\hline 7 & Ethanol:Water (85:15) & No & $47.20 \pm 0.99$ & $0.27 \pm 0.03$ & $0.060 \pm 0.01$ & $156.50 \pm 0.28$ & $517.65 \pm 13.79$ & $-51.2 \pm 1.41$ \\
\hline
\end{tabular}

${ }^{*}$ Each preparation suspended $3 \mathrm{mg}$ of TMZ and $30 \mathrm{mg}$ of copolymer in $3 \mathrm{~mL}$ of DMSO, which acted as the organic phase. The external phase contained $1 \%(w / v)$ Poloxamer 188.

${ }^{\wedge}$ Each experiment was repeated, and the data were expressed as mean $\pm \mathrm{SD}(n=2)$. 
In this method, DMSO/DCM and DMF/DCM mixed solvents were used as the dispersed phases of the drug and copolymer, and the two-phase dispersing phases contained TPGA that acted as the surfactant. The DL remained low at $0.2 \%$, and the particle sizes was approximately $470 \mathrm{~nm}$ (preparation 1, Table 3). Substituting DMSO with DMF and saturating the aqueous phase with sodium chloride did not improve the DL (Table 3). On the other hand, TMZ-loaded PLGA-PEG-FOL nanoparticles preparation using the nanoprecipitation method (Table 4) has resulted in 10 to 100 times reduction in the TMZ loading compared with that observed from the solvent evaporation methods. Besides that, there was an increase in the size of the TMZ-loaded nanoparticles. Nonetheless, saturating the external phase with sodium chloride improved the yield, EE, DL and the size of the nanoparticles (preparations 5 vs. 7, Table 4). In all the modified solvent emulsification methods and the nanoprecipitation method, the nanoparticle size increased tremendously after freeze-drying, as indicated by their size measured using the Zetasizer (Tables 3 and 4).

In the in vitro release study, we did not detect TMZ in the supernatant. But a peak, which was consistent to that of the AIC was detected, and the peak size increased gradually between $3 \mathrm{~h}$ and $24 \mathrm{~h}$. At $3 \mathrm{~h}$, the amount of AIC was $2.6 \mu \mathrm{g}$ and the amount doubled to $5 \mu \mathrm{g}$ only after $24 \mathrm{~h}$ (Figure 1 ). The AIC amount was equivalent to $8.5 \%$ and $16.7 \%$ of the amount of the TMZ used.

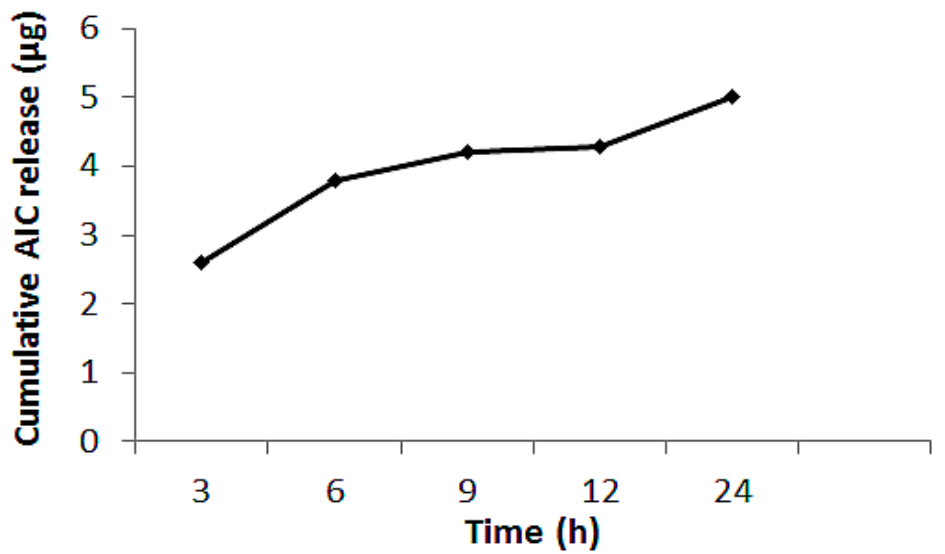

Figure 1. The release of the metabolic product of TMZ, 5-aminoimidazole-4-carboxamide (AIC) in vitro from the TMZ-loaded PLGA-PEG-FOL nanoparticles.

\section{Discussion}

Data from the first solvent evaporation method (from preparation 3, Table 1) suggested that when DCM was used as an organic solvent, high TMZ concentration may be required to achieve a better DL. At higher TMZ concentration, less drug is expected to diffuse out through the organic-aqueous interphase, i.e., from the organic phase into the dispersing phase. Ling et al. [16] showed that the use of DMF as an organic solvent and TPGS as the surfactant give a DL of between $0.15 \%$ and $17.95 \%$, and the DL increases in proportion to the amount of TMZ used, which is at the range between $10 \mathrm{mg}$ and $80 \mathrm{mg}$. DMF is a polar solvent that is able to dissolve TMZ, and TPGS is a derivative of vitamin E, thus is water soluble. TPGS is reported to have 67 times higher emulsification effects than PVA on PLGA-based nanoparticles $[17,18]$. However, residual PVA that clung to the nanoparticles has prevented dissolving of the nanoparticles for direct measurement of the DL using UV/Vis spectrophotometry technique. Therefore, in our subsequent preparation (Table 2), we increased the amount of TMZ by 10-fold (vs. preparation 1, Table 1) and substituted DCM with DMF, and replaced PVA with TPGS. However, our results were inconsistent with those reported previously [16].

From the solvent evaporation method, other study has shown that having $20 \mathrm{mg}$ of TMZ in the organic phase, and saturating the aqueous dispersing phase with TMZ gives a higher TMZ loading than that of an dispersing phase not saturated with TMZ (14\% vs. 0.4\%) [16]. However, this approach means that a large amount of TMZ will be required in preparing the nanoparticles, and this implies 
high cost and waste. We attempted a modified saturation method $[19,20]$. In this modified method, an emulsion was first generated when the organic phase was added to a smaller volume of the TPGS solution, and this was aimed to minimise the diffusion of TMZ under shear force during the second emulsification, leveraging also on the TPGS concentration gradient. By using a higher amount of TMZ, it may saturate the first TPGS solution when the primary emulsion is generated, hence may improve drug entrapment. The primary emulsion is then added to a larger volume of TPGS solution under stirring to encourage diffusion and evaporation of the organic solvent. We used solvent to dissolve or suspend TMZ as the drug has limited solubility in DCM. The ratios of the co-solvent used, as shown in Table 3, were the optimal combination.

Besides that, in preparation 3 (Table 3), the dispersing phase was comprised of TPGS dissolved in ethanol/water mixture saturated with sodium chloride. Because TMZ has lower solubility in ethanol than in water, we estimated that ethanol may reduce the diffusion of TMZ to the TPGS aqueous solution, and therefore give better entrapment of TMZ in the nanoparticles. Sodium chloride was used to saturate the TPGS solution so as to reduce the concentration gradient of the solute between the solvent and the aqueous phase, thereby reducing the diffusion potential and improves the encapsulation of TMZ [21,22]. Nonetheless, ethanol and sodium chloride have little effect on the loading efficiency as the preparation has comparably low DL as with other preparations (Table 3). It is unclear why sodium chloride did not improve the loading. It is worth noting that from preparations 1 to 3 , the primary emulsion formed large emulsion droplets when it was introduced to the subsequent TPGS solution, and the large emulsion droplets could only be dispersed with shear forces [23].

Nanoprecipitation method is widely used in studies involving hydrophilic drugs [21,24,25]. However, in this study, using either water or ethanol only as a dispersing phase did not improve the DL of TMZ (Table 4). A combination of ethanol and water with a ratio of 85 to 15 , and saturation of the mixture with sodium chloride led to an increase in EE and DL. Saturated ethanol and water solution, with ethanol and water prepared at different ratios did not give such effects. Overall, nanoprecipitation method did not compare favorably against solvent evaporation method in our preparation of TMZ loaded PLGA-PEG-FOL nanoparticles.

It was noted that, in all the solvent emulsification and nanoprecipitation methods studied, the nanoparticle size increased significantly to between 370-520 nm (Table 3) and 430-630 nm (Table 4) after freeze-drying. This was most likely due to particle aggregation under cryogenic stress condition. Cryo-protectant will be needed to minimise the particle agglomeration effect. In contrast, nanoparticles that were not subjected to free-drying cycle were generally less than $200 \mathrm{~nm}$.

In the in vitro release study, there are two main reasons as to why TMZ was not detected in the PBS-(1) TMZ is hydrolytically labile and most of the TMZ may have already been degraded the moment it was released to the medium; (2) Due to its degradation, TMZ, if present, has amount that was too little to be detected in the medium. Despite this, the cumulative profile of AIC suggested a sustained release of TMZ from the PLGA-PEG-FOL nanoparticles. This property of the nanoparticles is beneficial as it reduces the needs of frequent administration.

\section{Materials and Methods}

\subsection{General Information}

PLGA (molecular weight of 50,000 Dalton) was purchased from JenKem Technology (Plano, TX, USA), and PEG (molecular weight of 3500 Dalton) was supplied by DURECT Corporation (Birmingham, AL, USA). All the other chemicals and reagents were from Sigma (St. Louis, MO, USA). The solvents used were of analytical grade. NMR spectra were recorded on a Bruker $600 \mathrm{MHz}$ HNMR spectrometer in the University of Malaya, Kuala Lumpur. Deuterated dimethyl sulfoxide (DMSO-d6) was used as a solvent with tetramethysilane as an internal standard. Double beam UV-visible spectrophotometer (Perkin Elmer Lambda 25), a UV-Vis spectroscopy technique, was used to measure the drug loading and drug encapsulation efficiency, and in the drug release studies. 


\subsection{Preparation of PLGA-PEG-COOH}

PLGA-PEG was synthesised using a reported method [26]. Briefly, N-hydroxysuccinimide (NHS, $3 \mathrm{mg}, 0.026 \mathrm{mmol}$ ) and 1-ethyl-3-(3-dimethylaminopropyl)carbodiimide hydrochloride (EDC, $5 \mathrm{mg}$, $0.026 \mathrm{mmol}$ ) were dissolved in dichloromethane (DCM, $2 \mathrm{~mL}$ ), followed by addition of PLGA-COOH $(250 \mathrm{mg}, 0.005 \mathrm{mmol})$ dissolved in DCM $(2 \mathrm{~mL})$ with stirring. The resulting solution was then precipitated with diethyl ether-methanol $(20 \mathrm{~mL}, 50: 50, v / v \%)$, followed by centrifugation at $8000 \mathrm{~g}$ for $20 \mathrm{~min}$ to remove residual NHS and EDC. Centrifugation and washing with deionised water were repeated twice. The PLGA-NHS pellet was obtained and dried under vacuum to remove residual diethyl ether and methanol.

To prepare PLGA-PEG-COOH (Scheme 1), $\mathrm{NH}_{2}-\mathrm{PEG}-\mathrm{COOH}(20.4 \mathrm{mg}, 0.006 \mathrm{mmol}$ ) and $\mathrm{N}, \mathrm{N}$-diisopropylethylamine (DIEA, $7.5 \mathrm{mg}, 0.06 \mathrm{mmol}$ ) were added to a solution of PLGA-NHS (200 mg, $0.004 \mathrm{mmol})$ in DCM $(4 \mathrm{~mL})$ and then stirred for $24 \mathrm{~h}$ at room temperature. The resulting solution was precipitated with diethyl ether-methanol $(20 \mathrm{~mL}, 1: 1 ; v / v \%)$ solvent mixture and centrifuged at $8000 \mathrm{~g}$ for $20 \mathrm{~min}$ to remove the unreacted PEG and DIEA. Centrifugation and washing with deionised water were repeated twice. The PLGA-PEG-COOH pellet was thus obtained and dried under vacuum.

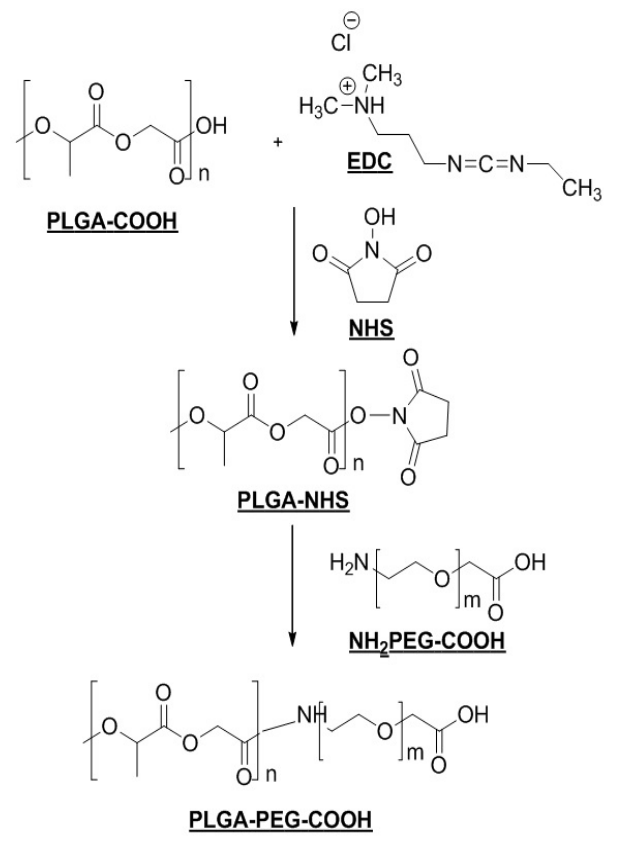

Scheme 1. Reaction scheme for the synthesis of PLGA-PEG-COOH from PLGA-COOH.

NHS (2.3 mg, $0.02 \mathrm{mmol})$ and EDC ( $4.0 \mathrm{mg}, 0.02 \mathrm{mmol})$ were dissolved in DCM (2 mL) and then added to PLGA-PEG-COOH $(200 \mathrm{mg}, 0.0037 \mathrm{mmol})$ solution in DCM $(4 \mathrm{~mL})$ with stirring for $60 \mathrm{~min}$ at room temperature. The resulting solution was precipitated with excess cold methanol and centrifuged at $8000 \mathrm{~g}$ for $20 \mathrm{~min}$ to remove the excess NHS and EDC. Centrifugation and washing were repeated twice. The PLGA-PEG-NHS obtained was dried under vacuum.

Folic acid was conjugated to PLGA-PEG-NHS by an amidation reaction (Scheme 2). In this preparation, PLGA-PEG-NHS ( $200 \mathrm{mg}, 0.0037 \mathrm{mmol}$ ) was dissolved in dimethyl sulfoxide (DMSO, $4 \mathrm{~mL}$ ). Folic acid (5-10 molar in excess) was added into the solution and stirred in the dark for $24 \mathrm{~h}$ at room temperature. The solution was precipitated with cold methanol $(20 \mathrm{~mL})$ and centrifuged at $8000 \mathrm{~g}$ for $20 \mathrm{~min}$. The precipitate, PLGA-PEG-FOL, was collected, dried under vacuum and then dissolved in DMSO ( $5 \mathrm{~mL}$ ) before it was placed in a dialysis bag and dialysed for $24 \mathrm{~h}$ against deionised water to remove unreacted folic acid. The cut-off molecular weight of the dialysis membrane was 11,295 Dalton. PLGA-PEG-FOL was characterised by ${ }^{1} \mathrm{H}-\mathrm{NMR}$ (DMSO-d6, ppm: 1.55, 3.41, 4.71-5.01, 
$6.64,6.90,7.65,7.87)$. Figure 2 shows the HNMR spectrum which confirmed the chemical structure of PLGA-PEG-FOL.

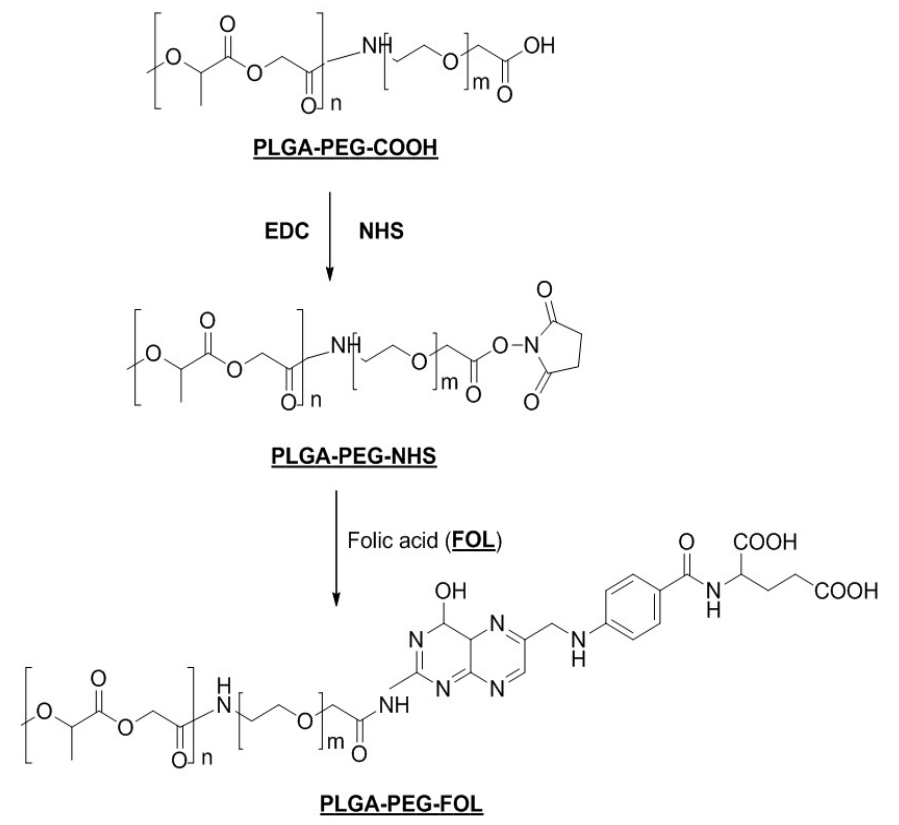

Scheme 2. Reaction scheme for the synthesis of PLGA-PEG-FOL.

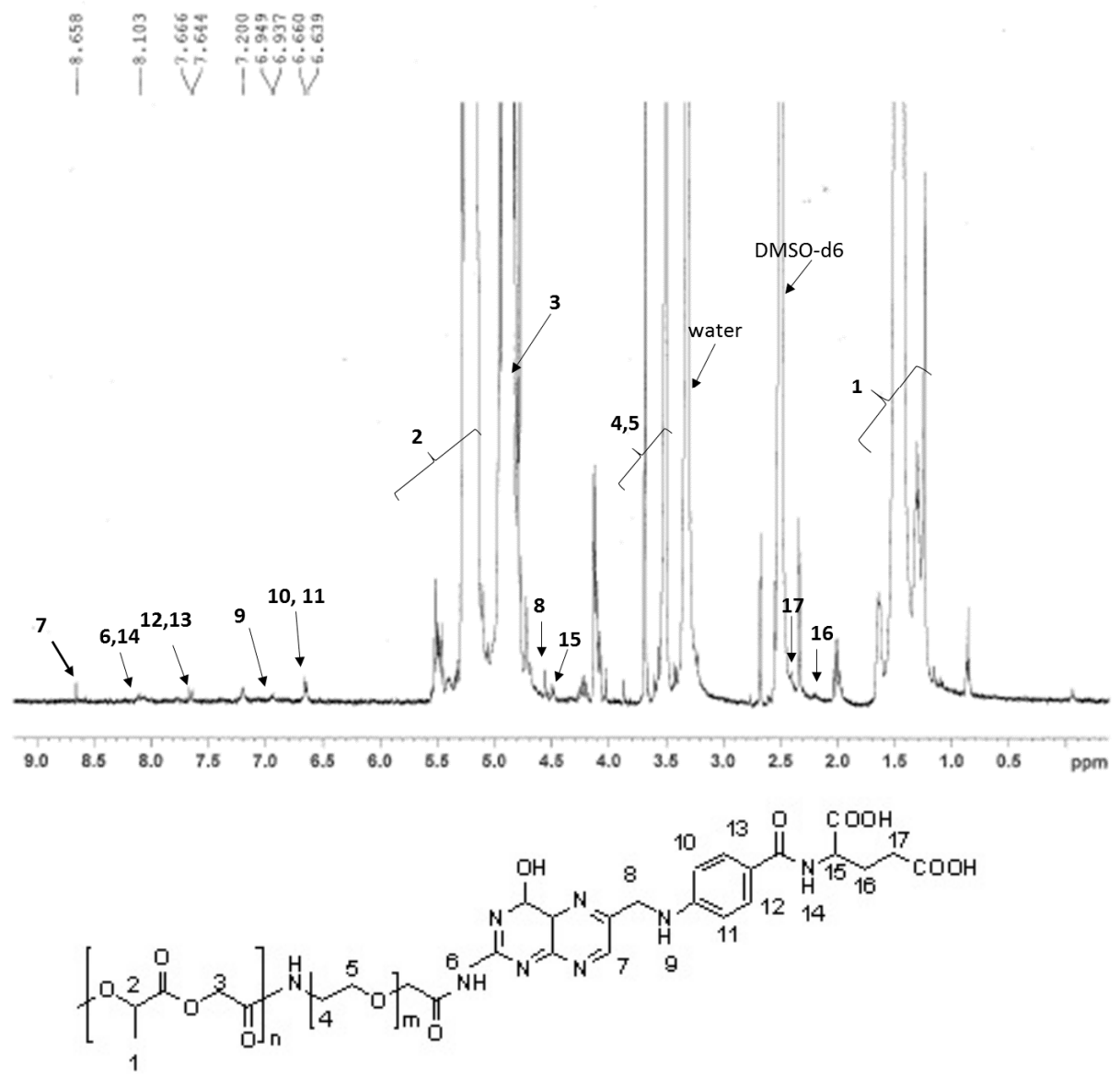

Figure 2. (Top) The NMR spectrum of PLGA-PEG-FOL. (Bottom) The numbering in the chemical structure of PLGA-PEG-FOL refers to the corresponding proton resonance peaks in the NMR spectrum. 


\subsection{Preparation of TMZ Loaded Nanoparticles by Using Emulsion Solvent Evaporation Method (1)}

Two milligrams of TMZ were dissolved in DCM $(4 \mathrm{~mL})$, and the solution was homogenised by sonication before addition of PLGA-PEG-FOL copolymer $(50 \mathrm{mg})$. The solution was then added slowly into $1 \%(w / v)$ polyvinyl alcohol (PVA, $20 \mathrm{~mL}$ ) aqueous solution and probe sonicated at $50 \mathrm{~W}$ for $1 \mathrm{~min}$ in an ice bath. The emulsion was stirred for $2 \mathrm{~h}$ and ultra-centrifuged at 10,000 rpm for $30 \mathrm{~min}$. The supernatant was removed after each centrifugation and the pellet, i.e., nanoparticles were washed three times with deionised water before being freeze-dried. The amount of TMZ encapsulated in the nanoparticles was calculated as the difference between the initial TMZ amount used and the TMZ recovered from the supernatant [27]. The amount of TMZ was quantified by $\mathrm{UV} /$ Vis spectrophotometry technique measured at $330 \mathrm{~nm}$, and drug loading (DL) was defined as the percentage of drug mass in the nanoparticles. Encapsulation efficiency (EE) was calculated as the percentage of actual drug mass encapsulated in the nanoparticles in relative to the initial amount of drug used [16]. The yield of the formulation was determined by calculating the difference between the total mass of nanoparticles after freeze-drying (M2) and the total mass of copolymer and TMZ used in preparing the formulation (M1) and from $(\mathrm{M} 2 / \mathrm{M} 1) \times 100 \%$.

\subsection{Preparation of TMZ Loaded Nanoparticles by Using Emulsion Solvent Evaporation Method (2)}

Experiments were conducted according to the procedures reported previously with some modification [16]. TMZ (20 mg) was dissolved in dimethylformamide (DMF, $1 \mathrm{~mL}$ ) before the addition of copolymer $(50 \mathrm{mg})$. The solution was slowly added into $0.05 \%(w / v) \mathrm{D}$ - $\alpha$-tocopheryl polyethylene glycol 1000 succinate (vitamin E TPGS, $25 \mathrm{~mL}$ ) aqueous solution and emulsified for $5 \mathrm{~min}$ in ice-bath using a micro-tip probe sonicator operated in pulse mode. The oil-in-water emulsion was stirred for $4 \mathrm{~h}$ at room temperature to allow diffusion of the organic solvent and solidification of the nanoparticles. The solution was ultra-centrifuged at 10,000 rpm for $20 \mathrm{~min}$ and the pellet washed three times with deionised water to remove the un-encapsulated TMZ. Nanoparticles were freeze-dried, and the lyophilised nanoparticles were dissolved in acetonitrile to determine the DL. The EE and the yield were measured as described above.

\subsection{Preparation of TMZ Loaded Nanoparticles by Using Emulsion Solvent Evaporation Method (3)}

The following procedures were modified from methods reported by Italia et al. [19] and Ling et al. [20]. Twenty milligrams of TMZ were dissolved in $2 \mathrm{~mL}$ of DMSO-DCM (1:3) or DMF-DCM (0.6:1.4), followed by the addition of PLGA-PEG-FOL copolymer (50 $\mathrm{mg})$. The solution was mixed with aqueous solution containing $0.2 \%(w / v)$ TPGS $(2 \mathrm{~mL})$ and emulsified for $5 \mathrm{~min}$ using a probe sonicator (output power of $50 \mathrm{~W}$ in pulse mode). The emulsion was then added to a second TPGS solution $(15 \mathrm{~mL}, 0.025 \%(w / v))$ placed under mechanical stirring at $600 \mathrm{rpm}$, and the oil-in-water emulsion was agitated for $4 \mathrm{~h}$ at room temperature. The suspension was centrifuged at 10,000 rpm for $20 \mathrm{~min}$, and the pellet washed with deionised water to remove residual drug and surfactant. The nanoparticles were freeze-dried, dissolved in acetonitrile, and the TMZ loading was determined by UV/Vis spectrophotometry.

\subsection{Preparation of TMZ Loaded Nanoparticles by Using the Nanoprecipitation Method}

According to this method, the polymer and the drug are dissolved in a solvent that is miscible with water. The polymer-drug solution is dispersed into the aqueous dispersing medium in which nanoparticles will be formed immediately when the solvent diffuses into the dispersing phase. Drug is entrapped when the polymer precipitated and solidified into nanoparticles $[21,24,25]$. Solutions of $15 \mathrm{~mL}$ of $1 \%(w / v)$ Poloxamer 188 in ethanol, water, and ethanol/water mixtures with varying proportion as dispersing media were prepared, respectively. Dispersing media saturated with sodium chloride were also prepared and evaluated. TMZ (3 mg) and PLGA-PEG-FOL copolymer (30 mg) were dissolved in DMSO $(3 \mathrm{~mL})$ and the DMSO solution was added dropwise $(0.3 \mathrm{~mL} / \mathrm{min})$ to the 
dispersing phase using a syringe (with a $25 \mathrm{G}$ needle) positioned above the medium, which was under moderate stirring. Stirring was continued for another $2 \mathrm{~h}$ to allow diffusion of the organic solvent. The resulting solution was centrifuged at 25,000 rpm for $30 \mathrm{~min}$ and the nanoparticles that appeared as the pellet were subjected to freeze-drying. The lyophilised nanoparticles were weighed and dissolved in acetonitrile, and the amount of encapsulated TMZ was determined by UV/Vis spectrophotometry.

\subsection{Characterisation of the Nanoparticles}

The particle size, size distribution and the surface charges (i.e., zeta potentials) of the nanoparticles were measured using a Nano-ZS Zetasizer equipped with a dynamic light scattering detector (Malvern Instruments, Malvern, UK).

\subsection{In Vitro Release Study}

We examined the release profile of TMZ from the nanoparticles that were generated from the solvent evaporation Method 3, as this method gave us a better yield compared to all the other methods. The release study was conducted as reported before with minor modification [16,28]. TMZ is hydrolytically labile, therefore its degradation product 5-aminoimidazole-4-carboxamide (AIC) was also measured [28]. Fifteen milligram of nanoparticles were suspended in phosphate-buffered saline (PBS, pH 7.4, $2 \mathrm{~mL}$ ) in a screw-capped tube and incubated in an orbital shaker at $37^{\circ} \mathrm{C}$. At the end of each time point $(3,6,9,12$ and $24 \mathrm{~h}$ ), the suspension was centrifuged at 10,000 rpm for $10 \mathrm{~min}$. The supernatant was collected and analysed for TMZ and AIC by UV/Vis spectrophotometry at $330 \mathrm{~nm}$ and $267 \mathrm{~nm}$, respectively. The absorbance wavelength of the AIC was determined by using the standard compound of AIC. The pellet was re-suspended in $2 \mathrm{~mL}$ of fresh PBS and incubation continued.

\section{Conclusions}

The methods used in this study are not new, but the idea of making TMZ loaded into PLGA-PEG-FOL nanoparticles, i.e., the combination of TMZ and PLGA-PEG-FOL has not been reported before. PLGA-PEG-FOL may allow targeted delivery of the drug, and the final product would be ideal for TMZ delivery. However, as the formulation attempts were not successful, we should consider finding a balance between being idealistic in our experimental design and what we can actually achieve in practice.

Our data suggested that neither the emulsion solvent evaporation method nor the nanoprecipitation method is suitable for our intended preparation. Both methods are commonly used in the preparation of polymeric nanoparticles. Despite the numerous and extensive modifications done on these methods, the percentage of drug loading was not satisfactory. Reflecting on the discrepancy between our data and with that reported in the literature, it appears that the amount of TMZ used in preparing TMZ nanoparticles, as well as the solubility of TMZ in the solvent used to dissolve the drug, are critical in achieving a high DL. The saturation method [16] gives a much improved DL, but it is only a 30 -fold increase in DL compared to a non-saturated condition. Thus, creating a simulated saturated condition may be a better alternative and is more cost effective.

The technical difficulties encountered in the present study could also be attributed to the loss of drug during the emulsification or precipitation step, where TMZ diffused out into the aqueous dispersing medium under high shear force. Unless these issues are resolved, the production of TMZ-PEG-PLGA-FOL nanoparticles using these two methods remains challenging. A more realistic solution here might be to explore new preparation method to obtain these nanoparticles. Despite having a relatively low DL, the release profile of the PLGA-PEG-FOL nanoparticles suggested that TMZ was released in a sustained manner, and this is indeed a positive outcome achieved in this study.

Acknowledgments: This study was supported by Monash University Malaysia and National Cancer Council Malaysia. We would like to thank You Zhuan Lee for conducting the experiments. 
Author Contributions: C.Y.L. and I.H.O. conceived and designed the experiments; Y.Z.L. performed the experiments; C.Y.L. and I.H.O. analysed the data and wrote the paper.

Conflicts of Interest: The authors declare no conflict of interest.

\section{References}

1. Stupp, R.; Mason, W.P.; van den Bent, M.J.; Weller, M.; Fisher, B.; Taphoorn, M.J.; Belanger, K.; Brandes, A.A.; Marosi, C.; Bogdahn, U.; et al. Radiotherapy plus concomitant and adjuvant temozolomide for glioblastoma. N. Engl. J. Med. 2005, 352, 987-996. [CrossRef] [PubMed]

2. Friedman, H.S.; Kerby, T.; Calvert, H. Temozolomide and treatment of malignant glioma. Clin. Cancer Res. 2000, 6, 2585-2597. [PubMed]

3. Payne, M.J.; Pratap, S.E.; Middleton, M.R. Temozolomide in the treatment of solid tumours: current results and rationale for dosing/scheduling. Crit. Rev. Oncol. Hematol. 2005, 53, 241-252. [CrossRef] [PubMed]

4. Thomas, R.P.; Recht, L.; Nagpal, S. Advances in the management of glioblastoma: The role of temozolomide and MGMT testing. Clin. Pharmacol. 2013, 5, 1-9. [PubMed]

5. Huang, G.; Zhang, N.; Bi, X.; Dou, M. Solid lipid nanoparticles of temozolomide: Potential reduction of cardial and nephric toxicity. Int. J. Pharm. 2008, 355, 314-320. [CrossRef] [PubMed]

6. Tian, X.H.; Lin, X.N.; Wei, F.; Feng, W.; Huang, Z.C.; Wang, P.; Ren, L.; Diao, Y. Enhanced brain targeting of temozolomide in polysorbate-80 coated polybutylcyanoacrylate nanoparticles. Int. J. Nanomedicine 2011, 6, 445-452. [PubMed]

7. Acharya, S.; Sahoo, S.K. PLGA nanoparticles containing various anticancer agents and tumour delivery by EPR effect. Adv. Drug Deliv. Rev. 2011, 63, 170-183. [CrossRef] [PubMed]

8. Kumari, A.; Yadav, S.K.; Yadav, S.C. Biodegradable polymeric nanoparticles based drug delivery systems. Colloids Surf. B Biointerfaces 2010, 75, 1-18. [CrossRef] [PubMed]

9. Dinarvand, R.; Sepehri, N.; Manoochehri, S.; Rouhani, H.; Atyabi, F. Polylactide-co-glycolide nanoparticles for controlled delivery of anticancer agents. Int. J. Nanomedicine 2011, 6, 877-895. [CrossRef] [PubMed]

10. Avgoustakis, K. Pegylated poly(lactide) and poly(lactide-co-glycolide) nanoparticles: Preparation, properties and possible applications in drug delivery. Curr. Drug Deliv. 2004, 1, 321-333. [CrossRef] [PubMed]

11. Wang, A.Z.; Gu, F.; Zhang, L.; Chan, J.M.; Radovic-Moreno, A.; Shaikh, M.R.; Farokhzad, O.C. Biofunctionalized targeted nanoparticles for therapeutic applications. Expert Opin. Biol. Ther. 2008, 8, 1063-1070. [CrossRef] [PubMed]

12. Saul, J.M.; Annapragada, A.; Natarajan, J.V.; Bellamkonda, R.V. Controlled targeting of liposomal doxorubicin via the folate receptor in vitro. J. Control. Release 2003, 92, 49-67. [CrossRef]

13. Oyewumi, M.O.; Yokel, R.A.; Jay, M.; Coakley, T.; Mumper, R.J. Comparison of cell uptake, biodistribution and tumor retention of folate-coated and PEG-coated gadolinium nanoparticles in tumor-bearing mice. J. Control. Release 2004, 95, 613-626. [CrossRef] [PubMed]

14. Kim, S.H.; Jeong, J.H.; Chun, K.W.; Park, T.G. Target-specific cellular uptake of PLGA nanoparticles coated with poly(L-lysine)-poly(ethylene glycol)-folate conjugate. Langmuir 2005, 21, 8852-8857. [CrossRef] [PubMed]

15. Zhao, H.; Yung, L.Y. Selectivity of folate conjugated polymer micelles against different tumor cells. Int. J. Pharm. 2008, 349, 256-268. [CrossRef] [PubMed]

16. Ling, Y.; Wei, K.; Zou, F.; Zhong, S. Temozolomide loaded PLGA-based superparamagnetic nanoparticles for magnetic resonance imaging and treatment of malignant glioma. Int. J. Pharm. 2012, 430, 266-275. [CrossRef] [PubMed]

17. Zhang, Z.; Tan, S.; Feng, S.S. Vitamin E TPGS as a molecular biomaterial for drug delivery. Biomaterials 2012, 33, 4889-4906. [CrossRef] [PubMed]

18. Guo, Y.; Luo, J.; Tan, S.; Otieno, B.O.; Zhang, Z. The applications of Vitamin E TPGS in drug delivery. Eur. J. Pharm. Sci. 2013, 49, 175-186. [CrossRef] [PubMed]

19. Italia, J.L.; Yahya, M.M.; Singh, D.; Ravi Kumar, M.N. Biodegradable nanoparticles improve oral bioavailability of amphotericin B and show reduced nephrotoxicity compared to intravenous Fungizone ${ }^{\circledR}$. Pharm. Res. 2009, 26, 1324-1331. 
20. Ling, Y.; Wei, K.; Luo, Y.; Gao, X.; Zhong, S. Dual docetaxel/superparamagnetic iron oxide loaded nanoparticles for both targeting magnetic resonance imaging and cancer therapy. Biomaterials 2011, 32, 7139-7150. [CrossRef] [PubMed]

21. Bilati, U.; Allemann, E.; Doelker, E. Development of a nanoprecipitation method intended for the entrapment of hydrophilic drugs into nanoparticles. Eur. J. Pharm. Sci. 2005, 24, 67-75. [CrossRef] [PubMed]

22. Peltonen, L.; Aitta, J.; Hyvonen, S.; Karjalainen, M.; Hirvonen, J. Improved entrapment efficiency of hydrophilic drug substance during nanoprecipitation of poly(I)lactide nanoparticles. AAPS PharmSciTech 2004, 5, 115-120.

23. Sahana, D.K.; Mittal, G.; Bhardwaj, V.; Kumar, M.N. PLGA nanoparticles for oral delivery of hydrophobic drugs: influence of organic solvent on nanoparticle formation and release behavior in vitro and in vivo using estradiol as a model drug. J. Pharm. Sci. 2008, 97, 1530-1542. [CrossRef] [PubMed]

24. Barichello, J.M.; Morishita, M.; Takayama, K.; Nagai, T. Encapsulation of hydrophilic and lipophilic drugs in PLGA nanoparticles by the nanoprecipitation method. Drug Dev. Ind. Pharm. 1999, 25, 471-476. [CrossRef] [PubMed]

25. Redhead, H.M.; Davis, S.S.; Illum, L. Drug delivery in poly (lactide-co-glycolide) nanoparticles surface modified with poloxamer 407 and poloxamine 908: In vitro characterisation and in vivo evaluation. J. Control. Release 2001, 70, 353-363. [CrossRef]

26. Farokhzad, O.C.; Cheng, J.; Teply, B.A.; Sherifi, I.; Jon, S.; Kantoff, P.W.; Richie, J.P.; Langer, R. Targeted nanoparticle-aptamer bioconjugates for cancer chemotherapy in vivo. Proc. Natl. Acad. Sci. USA 2006, 103, 6315-6320. [CrossRef] [PubMed]

27. Betancourt, T.; Brown, B.; Brannon-Peppas, L. Doxorubicin-loaded PLGA nanoparticles by nanoprecipitation: preparation, characterization and in vitro evaluation. Nanomedicine 2007, 2, 219-232. [CrossRef] [PubMed]

28. Lanz-Landazuri, A.; García-Alvarez, M.; Portilla-Arias, J.; de Ilarduya, A.M.; Holler, E.; Ljubimova, J.; Muñoz-Guerra, S. Modification of microbial polymalic acid with hydrophobic amino acids for drug-releasing nanoparticles. Macromol. Chem. Phys. 2012, 213, 1623-1631. [CrossRef] [PubMed]

(C) 2016 by the authors; licensee MDPI, Basel, Switzerland. This article is an open access article distributed under the terms and conditions of the Creative Commons Attribution (CC-BY) license (http://creativecommons.org/licenses/by/4.0/). 